\title{
Robotic total mesorectal excision for the treatment of rectal cancer
}

\author{
Seung Hyuk Baik • Chang Moo Kang • Woo Jung Lee • \\ Nam Kyu Kim • Seung Kook Sohn • Hoon Sang Chi • \\ Chang Hwan Cho
}

Received: 20 October 2006 / Accepted: 15 January 2007 / Published online: 10 February 2007

(C) Springer London 2007

\begin{abstract}
Robotic techniques have been developed to facilitate endoscopic surgery and to overcome its disadvantages. Thus, we performed robotic total mesorectal excison (TME) in a patient with rectal cancer, using the da Vinci ${ }^{\circledR}$ Surgical System. To our knowledge, this is the first robotic low anterior resection, based on standard TME principles, with pelvic autonomic nerve preservation. In conclusion, this robotic system is an excellent instrument for performing the standard TME procedure in rectal cancer patients.
\end{abstract}

Keywords Robotic surgery $\cdot$ Total mesorectal excision $\cdot$ Rectal cancer

\section{Introduction}

The da Vinci ${ }^{\circledR}$ surgical system (Intuitive Surgical, Sunnyvale, CA, USA) was developed specifically to compensate for the technical limitations of laparoscopic instruments, such as two-dimensional vision, misalignment of hands and instruments, limited dexterity of

This work was supported by Yonsei University Research Fund of 2006 (No. 6-2006-0111).

S. H. Baik · C. M. Kang · W. J. Lee

Robotic and Laparoscopic Surgery Center,

Severance Hospital, Seoul, Korea

S. H. Baik · C. M. Kang · W. J. Lee $(\bowtie) \cdot$ N. K. Kim •

S. K. Sohn · H. S. Chi - C. H. Cho

Department of Surgery, Yonsei University College

of Medicine, 134 Shinchon-dong, Seodaemun-ku,

C.P.O. Box 8044, 120-752 Seoul, Korea

e-mail:wjlee@yumc.yonsei.ac.kr instruments inside the patient, and fixed instrument tips. The da Vinci ${ }^{\circledR}$ system provides a stable camera platform, three-dimensional imaging, excellent ergonomics, tremor elimination, ambidextrous capability, motion scaling, and instruments with multiple degrees of freedom. However, until now, experience with robotic colorectal cancer surgery was limited worldwide, and there was no previous description of its use in total mesorectal excision (TME) with pelvic autonomic nerve preservation for rectal cancer patients [1$4,6,7]$. Therefore, herein, we describe our experience using robotic TME in a rectal cancer patient.

\section{Case report}

A 59-year-old female was referred by her general practitioner to our Robotic and Laparoscopic Surgery Center for further evaluation and management of a rectal mass. The patient had a five-year history of hypertension but had no other medical history.

The rectal mass was detected at $10 \mathrm{~cm}$ from the anal verge upon rigid sigmoidscopy. Total colonoscopy showed a fungating $\left(1.5 \times 1.5 \mathrm{~cm}^{2}\right)$ mass at the rectum with normal findings at other sites. Tattooing was done below the mass. Colonoscopic biopsy was performed, and the pathological diagnosis was moderately differentiated adenocarcinoma. Pre-operative MRI showed a $1.5-\mathrm{cm}$ fungating lesion in the mid-rectum but no evidence of mesorectal fat extension (Fig. 1). A pre-operative abdomino-pelvic computerized tomography (CT) scan showed no distant metastasis.

We decided to perform curative resection, using the da Vinci ${ }^{\circledR}$ surgical system (Intuitive Surgical). A standard mechanical bowel preparation was performed 


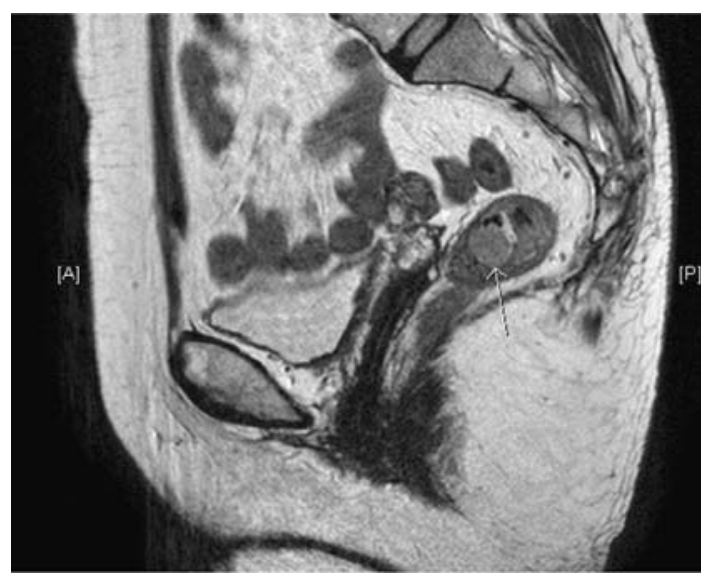

Fig. 1 Pre-operative MRI showed a 1.5-cm fungating lesion in the mid-rectum (arrow) but no evidence of mesorectal fat extension

$24 \mathrm{~h}$ prior to the operation, and a pneumatic compression stocking was applied $1 \mathrm{~h}$ prior to the operation.

The patient was placed supine with the legs apart in a $30^{\circ}$ Trendelenburg position with a $15^{\circ}$ right down. A 12-mm trocar was placed through an incison just above the umbilicus after achieving pneumoperitoneum; then, a $30^{\circ}$ standard $12-\mathrm{mm}$ robotic laparoscope was inserted through the $12-\mathrm{mm}$ trocar. The other two 8 -mm da Vinci ${ }^{\circledR}$ trocars were placed under direct visualization in the left MacBurney area on the lateral margin of the abdominal rectus muscle and the right MacBurney area, respectively. The 5-mm trocar was placed in the right midabdomen lateral to the umbilicus in the midaxillary line to allow assistant access for mobilization of the left colon. The 12-mm trocar was placed on the right, lateral to the right $8-\mathrm{mm}$ da Vinci ${ }^{\circledR}$ torcar to allow the initial mobilization of the left colon and the use of an endoscopic stapler. Mobilization of the left colon was undertaken, using standard laparoscopic instruments, after the inferior mesenteric artery and vein were divided proximally, using clips. Then, the robotic instrument was positioned between the legs. The rectum was suspended, using an atraumatic grasper through the left port, and rectal dissection in the mesorectal plane proceeded in front of the levator ani muscle, using the cautery hook in accordance with the TME principles (Fig. 2). The mesorectum was divided precisely at $2 \mathrm{~cm}$ beyond the tumor, using both robotic arms. The rectum itself was divided, using endo-GIA. The robotic instrument was then disengaged. A small transverse incision was made in the left iliac fossa. The specimen was retrieved, and the descending colon was then divided. The reconstruction was performed, using an EEA stapler. The pathologic diagnosis was moderately differentiated adenocarcinoma, and the main tumor had invaded the submucosa without lymph node metastasis in 23 regional lymph nodes. The quality of the mesorectum was complete grade, as defined in the Dutch TME trial (Fig. 3) [8]. The pelvic autonomic nerve was wellpreserved, and postoperative sexual and voiding functions were intact with no change between the preoperative and postoperative periods. The patient was discharged at seven days after the operation without complications.
Fig. 2 Robotic TME procure. a Right lateral dissection at the sacral promontory. b Posterior dissection between the presacral fascia and the rectal proper fascia. c Anterior dissection between the vagina (arrow) and the rectal anterior wall. d Posterior dissection in front of the levator ani muscle
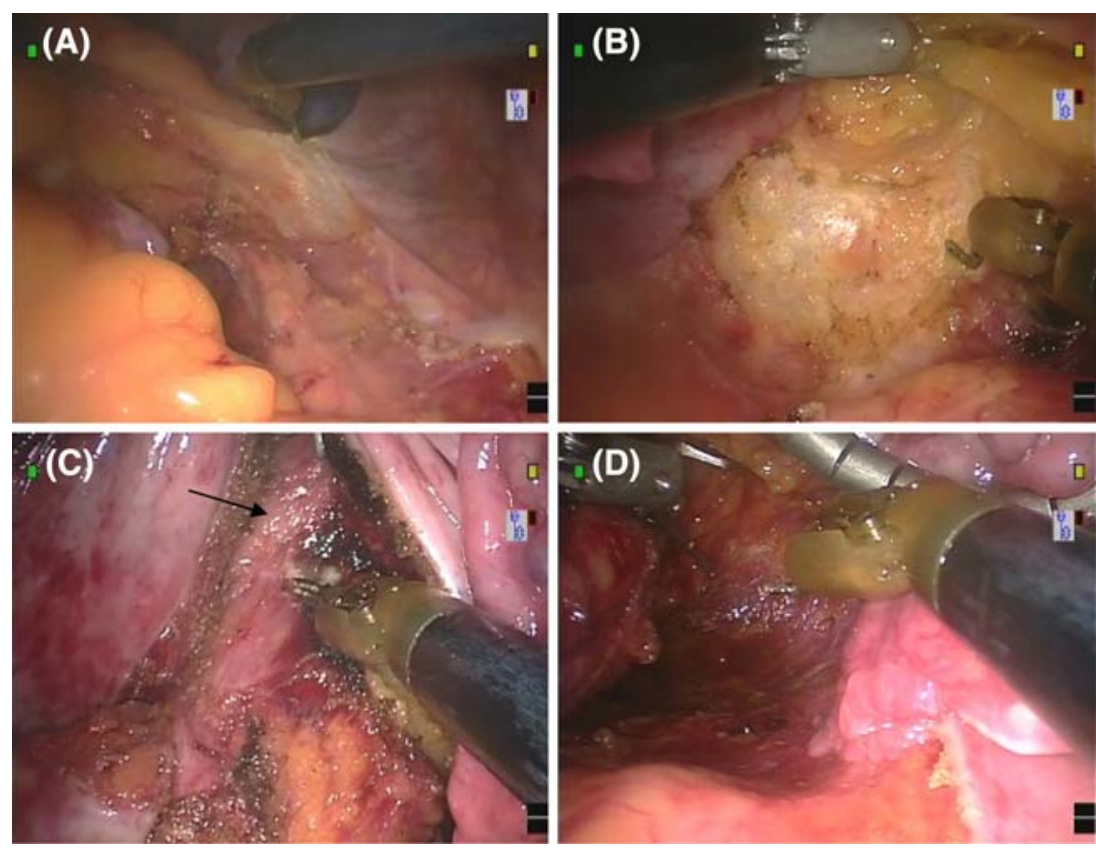


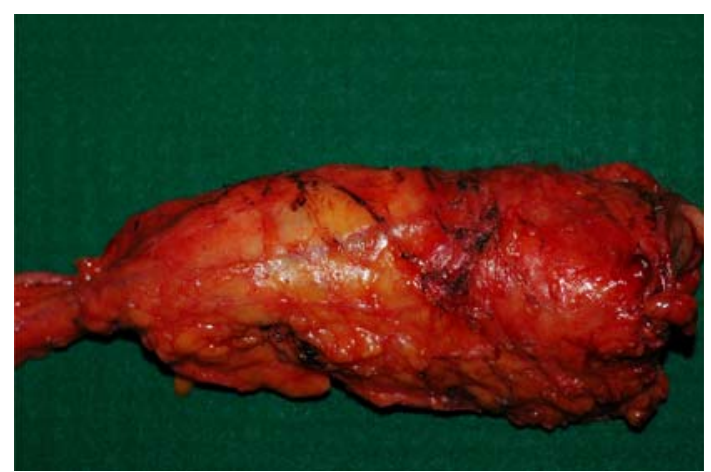

Fig. 3 Gross specimen finding. The quality of the mesorectum was complete grade

\section{Discussion}

In 1979, the procedure that is now known as total mesorectal excision (TME) was introduced [9]. It is now generally accepted as the gold standard for treatment of rectal cancer. Since the first laparoscopic colectomy in 1991, the use of laparoscopic surgery for colorectal cancer has been increasing, and the early results are highly encouraging $[10,11]$. Moreover, successful laparoscopic TME has been introduced with several advantages, when compared with open TME, including reduced postoperative pain, faster recovery of bowel function, and decreased hospital stay and disability [12, 13]. However, laparoscopic surgery has technical limitations, such as a lack of three-dimensional view, limited dexterity of the instruments inside the patient, and fixed instrument tips [14].

The da Vinci ${ }^{\circledR}$ surgical system (Intuitive Surgical) is modeled after the human wrist, with the cable system designed to be similar to the ligaments of the human wrist. Also, the da Vinci ${ }^{\circledR}$ system transposes fingers to the instrument tips, eliminates hand tremor, has ambidextrous capability, and can scale motion. These advantages can be applied in the field of colorectal surgery, and D'Annibale et al. [7] reported 52 cases that underwent robotic colorectal surgery. These authors concluded that the da Vinci ${ }^{\circledR}$ system may be useful in surgical procedures, such as splenic flexure takedown, dissection of the inferior mesenteric artery with identification of the nervous plexus, and dissection of a narrow pelvis. In our case, we performed dissection of the pelvis, using the da Vinci ${ }^{\circledR}$ system, and our conclusion is in agreement with D'Annibale et al. [7] with regard to pelvic dissection. However, there have not been previous reports of TME using a robotic system. In this case, we have performed standard TME with the help of dexterous robotic instrument tips, and the quality of the mesorectum was complete grade. This procedure was developed while keeping in mind the importance of complete excision, using direct vision, of the rectal proper fascia surrounding the mesorectum. Thus, TME can be performed more easily and effectively using the da Vinci ${ }^{\circledR}$ System.

Until now, for resection of the rectum, two steps have been necessary for the robotic system's placement because the robotic arms of the da $\mathrm{Vinci}^{\circledR}{ }^{\circledR}$ system are unable to self-adjust around the bed to allow the surgeon to gain access to more than one quadrant of the abdominal cavity [15]. The first placement is for the dissection of the left colon and sigmoid colon and to mobilize the spleen flexure. The second placement is for the dissection of the rectum and, for this, the da Vinci ${ }^{\circledR}$ system has to be moved to the coxa area. However, moving the da Vinci ${ }^{\circledR}$ system is a time-consuming and difficult procedure because the robotic devices are heavy and bulky. In our case, we used conventional laparoscopic instruments for the first step and the da Vinci ${ }^{\circledR}$ system for the second step. This hybrid procedure facilitated the whole procedure more effectively.

The major drawbacks of robotic systems are high cost, and a lack of tactile sensation and tensile feedback to the surgeon, who must depend on visual cues to estimate the tension exerted on tissue by the robotic arms. Therefore, the pre-sacral venous plexus can potentially be damaged during manipulation, causing profound hemorrhaging. However, in this case, we found that the lack of tactile sensation could be overcome by using effective visual cues during TME.

In conclusion, TME was performed safely and effectively, using the da Vinci ${ }^{\circledR}$ surgical system, because this system has more dexterity and flexibility than conventional laparoscopic instruments. However, prospective randomized studies are necessary to evaluate the preservation of sexual and voiding function, as well as the oncological outcomes of this approach.

\section{References}

1. Weber PA, Merola S, Wasielewski A et al (2002) Telerobotic-assisted laparoscopic right and sigmoid colectomies for benign disease. Dis Colon Rectum 45:1689-1694

2. Giulianotti PC, Coratti A, Angelini M et al (2003) Robotics in general surgery: personal experience in a large community hospital. Arch Surg 138:777-784

3. Vibert E, Denet C, Gayet B (2003) Major digestive surgery using a remote controlled robot: the next revolution. Arch Surg 138:1002-1006

4. Corcione F, Esposito C, Cuccurullo D et al (2005) Advantages and limits of robot-assisted laparoscopic surgery: preliminary experience. Surg Endosc 19:117-119

6. Woeste G, Bechstein WO, Wullstein C (2005) Does telerobotic assistance improve laparoscopic colorectal surgery? Int J Colorectal Dis 20:253-257 
7. D'Annibale A, Morpurgo F, Fiscon V et al (2004) Robotic and laparoscopic surgery for treatment of colorectal disease. Dis Colon Rectum 47:2162-2168

8. Nagtegaal ID, van de Velde CJ, van der Worp E et al (2002) Cooperative clinical investigators of the Dutch Colorectal Cancer Group. Macroscopic evaluation of rectal cancer resection specimen: clinical significance of the pathologist in quality control. J Clin Oncol 20:1729-1734

9. Heald RJ (1979) A new approach to rectal cancer. Br J Hosp Med 22:277-281

10. Fielding GA, Lumley J, Nathanson L et al (1997) Laparoscopic colectomy. Surg Endosc 11:745-749

11. Hoffman GC, Baker JW, Doxey JB et al (1996) Minimally invasive surgery for colorectal cancer: initial follow-up. Ann Surg 223:790-798
12. Kim SH, Milsom JW, Gramlich TL et al (1998) Does laparoscopic vs. conventional surgery increase exfoliated cancer cells in the peritoneal cavity during resection of colorectal cancer? Dis Colon Rectum 41:971-978

13. Schwander O, Schiedeck TH, Killaitis C et al (1999) A casecontrol-study comparing laparoscopic versus open surgery for rectosigmoidal and rectal cancer. Int $\mathbf{J}$ Colorectal Dis 14:158-163

14. Ballantyne GH (2002) Robotic surgery, telerobotic surgery, telepresence, and telemonitoring. Review of early clinical results.Surg Endosc 16:1389-402

15. Hanly EJ, Talamini MA (2004) Robotic abdominal surgery. Am J Surg 188:19S-26S 